\title{
Belgeo
}

Belgeo Revue belge de géographie

$1 \mid 2018$

Miscellaneous

\section{Changements environnementaux et migration en Afrique de l'Ouest. Une revue des études de cas}

\section{Loïc Brüning et Etienne Piguet}

\section{(2) OpenEdition}

1 Journals

\section{Édition électronique}

URL : http://journals.openedition.org/belgeo/28836

DOI : 10.4000/belgeo.28836

ISSN : 2294-9135

Éditeur :

National Committee of Geography of Belgium, Société Royale Belge de Géographie

Référence électronique

Loïc Brüning et Etienne Piguet, « Changements environnementaux et migration en Afrique de l'Ouest. Une revue des études de cas », Belgeo [En ligne], 1 | 2018, mis en ligne le 29 novembre 2018, consulté le 15 janvier 2020. URL : http://journals.openedition.org/belgeo/28836 ; DOI : 10.4000/belgeo.28836

Ce document a été généré automatiquement le 15 janvier 2020.

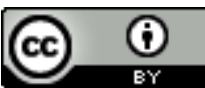

Belgeo est mis à disposition selon les termes de la licence Creative Commons Attribution 4.0 International. 


\title{
Changements environnementaux et migration en Afrique de l'Ouest. Une revue des études de cas
}

\author{
Loïc Brüning et Etienne Piguet
}

\section{Introduction}

1 La question des migrations que pourrait induire le changement climatique (CC) fait l'objet de recherches depuis une trentaine d'années et le nombre d'études de cas s'est fortement accru récemment (Guélat et al., 2016). On s'accorde à considérer l'Afrique subsaharienne comme tout particulièrement exposée au $\mathrm{CC}$ et à ses conséquences (IPCC, 2014). Trois synthèses de littérature permettent de faire un premier bilan des acquis de la recherche sur le continent et démontrent la complexité du lien environnement-migration.

Deux premières revues de littératures s'intéressent à l'Afrique subsaharienne dans son entièreté. Jonsson (2010) arrive ainsi à la conclusion que les stress environnementaux ne produisent pas nécessairement des migrations. Migrer demande passablement de ressources, par conséquent, des stratégies d'adaptation non-migratoires sont plus fréquemment préférées. Si une migration a lieu, elle est le plus souvent interne au pays et temporaire. En conséquence, les départs ne sont pas généralement soudains mais progressifs. Morrissey (2014) souligne quant à lui que le lien mobilité - environnement n'est pas nouveau et que la migration est une stratégie coutumière de longue date pour sécuriser les moyens de subsistance en Afrique subsaharienne. Quelques limites typologiques de ces deux revues de littérature peuvent être mises en lumière. Jonsson conceptualise le paysage de la recherche entre les études tenant du «push-pull » et les études «multi-level». Cette conceptualisation tend à cristalliser une frontière entre les deux approches qui n'est pas fermée mais au contraire perméable. En effet, certaines études tenantes $\mathrm{du}$ « push-pull » proposent de relativiser la relation déterministe entre l'environnement et la migration. A cet égard, Morrissey propose une autre typologie de 
la recherche orientée vers la méthodologie. Les études de cas sont donc classées par aléa. Bien que cette répartition permette de mettre en exergue les principaux résultats, elle peut avoir le désavantage d'obscurcir les aspects théoriques des études de cas exposées. Néanmoins, les deux auteurs se retrouvent dans leur conclusion car ils avancent que les liens entre environnement et migration sont complexes et qu'ils agissent à différents niveaux. Mais aussi, les deux auteurs suggèrent une ouverture de la recherche que cela soit d'un point de vue thématique ou méthodologique.

3 La synthèse de Van der Land et al. (2018) analyse quinze études de cas menées exclusivement en Afrique de l'Ouest. Les auteurs présentent les principales tendances et constats de la recherche mais ne proposent pas de typologie. Aussi, il est souligné que la grande variété de méthodes et de concepts utilisés rend donc difficile la comparaison des résultats même si dans la région observée les conditions environnementales et les aléas climatiques sont assez semblables. Néanmoins, il est mis en lumière l'importance de la migration circulaire et des conséquences des remises sur les capacités d'adaptation et sur la résilience. Les facteurs environnementaux ne sont donc pas les seules variables à étudier dans la prise de décision de migrer. Les futures recherches doivent alors utiliser des approches plus larges qui prennent en compte l'hétérogénéité des contextes ouest-africains.

4 Le présent article vise à apporter une plus-value à plusieurs niveaux par rapport aux synthèses existantes. Premièrement, nous présentons exclusivement les études de cas qui tentent d'expliquer les relations causales entre le changement climatique ou les dégradations environnementales et les migrations en Afrique de l'Ouest. Les nombreux articles traitant des conséquences au sens large des mouvements migratoires ne sont pas pris en compte. Deuxièmement, nous actualisons l'inventaire des études jusqu'en 2017 et couvrons ainsi de nouvelles études importantes, permettant d'approfondir considérablement l'analyse. Nous présentons ainsi quarante-trois études de cas en termes de lieux d'études, de méthodes utilisées, de concepts mobilisés et de résultats obtenus. Cette revue de littérature a donc l'avantage d'exposer une vue d'ensemble détaillée de la recherche que cela soit au niveau méthodologique, conceptuel, géographique et empirique. Pour ce faire, nous avons utilisé un outil de recherche développé par l'Institut de Géographie (IGG) de l'Université de Neuchâtel: Climig Database. Il s'agit d'une base de données en ligne, mise à jour hebdomadairement, qui recense toutes les publications qui traitent des migrations liées à l'environnement (cf. encadré).

5 Nous proposons une typologie des études en commençant par les travaux qui, sans mesurer l'impact de l'environnement sur les migrations, se contentent d'identifier des zones à risque où des migrations pourraient avoir lieu dans le futur. Nous opérons ensuite une distinction entre les résultats qui tendent à établir un impact univoque des changements environnementaux ${ }^{1}$ sur les migrations, et ceux qui, au contraire, relativisent cet impact. Nous évoquons ensuite les études qui proposent des interprétations multifactorielles et globales. Cette typologie reprend la proposition de Piguet (2010). Finalement, nous concluons en exposant les degrés de consensus en regard de dix résultats majeurs de la recherche.

6 En lien avec la typologie proposée, cette revue de littérature plaide en faveur d'une approche plurielle et compréhensive pour étudier les migrations environnementales en Afrique de l'Ouest. Comme le montre Piguet (2010) l'enjeu des recherches scientifiques est de dégager le poids exact du facteur environnemental dans la décision de migrer. 
Pourtant, il est difficile de mesurer l'influence de l'environnement dans la décision de migrer car cette dernière dépend de l'interaction entre les différents facteurs contextuels et également des types d'aléas climatiques (slow on-set events et rapid on-set events). De plus, les réponses aux stress environnementaux sont plurielles et composites $^{2}$. Les mouvements migratoires peuvent s'étendre sur des courtes ou de longues distances, de manière permanente ou temporaire et les déplacements peuvent être internes à un pays ou internationaux (Piguet et al., 2010a). Par conséquent, les résultats et les informations disponibles sont hétérogènes et morcelés en raison du grand nombre d'études de cas et d'une grande amplitude méthodologique (Ibid). De ce fait, on assiste à un développement théorique limité pour mesurer le rôle et le poids du facteur environnemental dans la migration humaine.

Une solution à cet obstacle théorique et méthodologique est de mieux intégrer la migration dans un contexte de dégradation environnementale avec les approches classiques de la migration. Cependant, les modèles classiques sont difficilement applicables à l'Afrique de l'Ouest comme le montrent Piché et Cordell (2015). En particulier, les modèles classiques ne prennent pas en compte la variable du retour, c'est-à-dire l'aspect circulaire de la migration. Ils supposent donc qu'un migrant va s'installer définitivement dans son nouveau lieu de vie. Néanmoins, le retour est une caractéristique de la migration ouest-africaine. Pour comprendre les migrations dans leur ensemble dans un contexte de changement environnemental, il est alors primordial de tenir compte des caractéristiques spatiales, sociales et économiques de la mobilité. Les recherches doivent donc s'orienter vers des approches plus compréhensives comme nous le proposons dans la conclusion. L'objectif n'est donc pas de rentrer dans une démarche explicative mais plutôt d'appréhender le phénomène des migrations environnementales d'une manière holistique.

\section{Encadré 1}

\section{CLIMIG Database : Méthodes de recherche et critères d'inclusion}

CLIMIG Database fonctionne avec quatre familles de mots-clés.

Area identifie les aires géographiques des études basées sur les définitions de l'ONU.

Les continents, les pays, les régions et les villes sont désignés dans ce champ de mots-clés.

Type identifie le genre d'étude de cas et sa méthodologie.

Overview est utilisé pour les synthèses sur le sujet. Law se rapporte aux recherches qui s'intéressent aux questions juridiques et politiques.

Pour les études empiriques, CLIMIG utilise le mot-clé type.

Type 1 : Modèles écologiques basés sur les caractéristiques d'une aire (analyse spatiale).

Type 2 : Analyse multi-niveaux basée sur les caractéristiques d'une aire et de l'individu. 
Type 3 : Analyse de données individuelles basée sur une enquête à large échantillon $(>100)$.

Type 4 : Analogues historiques.

Type 5 : Index de vulnérabilités, identification de hotspots, scénarios, études de cas régionales, etc.

Type 6 : Etudes de cas qualitatives utilisant des méthodes ethnographiques et des questionnaires à petits échantillons.

Environmental hazard identifie le type de perturbation environnementale étudiée.

Flood : inondation, inondation éclair, variation pluviométrique.

Hurricane : typhon, cyclone, tempête.

SLR : montée du niveau de la mer, érosion côtière.

Drought : désertification, vague de chaleur, dégradation des sols, diminution des précipitations.

Focus se rapporte à l'approche spécifique de la recherche.

DRR/EWS: disaster risk reduction/Early warning system

Gender : études qui traitent des migrations environnementales avec un focus sur les questions de genres.

Migadapt : utilisation de la migration comme une réponse adaptative ou comme une stratégie pour faire face aux dégradations environnementales.

Perception : représentations et perceptions du changement climatique des acteurs.

Statelessness : des situations d'apatride en raison du changement climatique.

Trapped : études traitant de l'immobilité et des populations piégées.

Conflict : études faisant le lien entre les migrations environnementales et les conflits

\section{Identification des zones à risque (hot-spots)}

Le continent africain est globalement le plus vulnérable face aux changements climatiques et ceci en raison de son haut degré d'exposition et de ses ressources financières restreintes pour l'adaptation. Il est particulièrement sensible aux changements de température et aux changements de pluviométrie tout comme aux aléas environnementaux soudains tels que les inondations (IPCC, 2014). L'Afrique de l'Ouest et le Sahel sont des territoires tout particulièrement identifiés comme "hotspots » pour plusieurs raisons. Il a été remarqué une tendance à une diminution des journées et des nuits froides et une augmentation des journées et des nuits chaudes 
(Ibid). Par conséquent, il y a eu un réchauffement de $0,5 \mathrm{C}^{\circ}$ et $0,8 \mathrm{C}^{\circ}$ entre 1970 et 2010 (Ibid). Également, on remarque une nouvelle distribution spatiale et une diminution des espèces ligneuses et des espèces herbacées. En effet, la savane a tendance à descendre vers le sud et ainsi empiéter sur la forêt tropicale (Ibid). Ce phénomène est notamment dû à la déforestation et à la désertification mais est aussi causé par des changements continus dans les régimes de précipitation et des températures.

Dans ce contexte, Müller et al. (2014), grâce à l'utilisation de quarante scénarios climatiques, définissent les « hot-spots» comme des régions de l'Afrique subsaharienne qui seront touchées sévèrement par le changement climatique tout en connaissant une forte densité de population ainsi qu'un haut taux de pauvreté. Neumann et al. (2015), considèrent à travers une analyse statistique spatiale à large échelle que les variations pluviométriques, l'aridité, la sécheresse, la dégradation des sols et ses contraintes ainsi que les disponibilités en terres cultivables et pâturables, sont les principaux aléas environnementaux qui génèrent des migrations dans les régions arides.

D'autres auteurs se concentrent sur des zones spécifiques. Il apparaît ainsi que, si les dégradations et les changements environnementaux accentuent les migrations vers les zones urbaines (Pedersen, 1995 ; Dillon et al., 2011), ces dernières sont également des zones à risques. Doussou et Glehouenou-Doussou (2007) analysent ainsi la vulnérabilité socioéconomique et biophysique de la ville de Cotonou face à la montée des eaux. Ces auteurs estiment, grâce à une simulation, que d'ici 2050 le niveau de la mer pourrait monter de sept à vingt centimètres et d'ici 2100 de vingt à quarante-neuf centimètres et par conséquent engendrer des flux migratoires. Cette recherche laisse sous-entendre que malgré l'attraction des centres urbains côtiers, ces derniers pourraient devenir des zones de départ en raison de la montée tendancielle du niveau des océans.

12 Les études qui se contentent d'évaluer l'intensité du risque restent relativement peu nombreuses en Afrique de l'Ouest en comparaison avec d'autres régions (Guélat et al., 2016). Une majorité d'auteurs semblent considérer le continent dans son ensemble comme vulnérable et approfondissent les analyses vers une évaluation de l'ampleur des conséquences migratoires comme l'illustrent les chapitres qui suivent.

\section{L'environnement a un impact confirmé sur les migrations}

13 Plusieurs recherches confirment l'impact des conditions environnementales sur les migrations en Afrique de l'Ouest. Ce sont principalement la pluviométrie et l'augmentation des températures ainsi que la dynamique du couvert végétal qui influencent de manière marquée les migrations internes ou inter-régionales et cela à plusieurs niveaux. Il apparaît également que les choix des destinations sont influencés par l'environnement. Si principalement les zones urbaines semblent être préférées aux zones rurales (Pedersen, 1995 ; Dillon et al., 2011), dans certaines conditions spécifiques ce n'est pas le cas. Finalement, les aléas environnementaux peuvent augmenter la migration sur des courtes distances mais ils peuvent aussi simultanément réduire les flux migratoires internationaux. 


\section{Les migrations internes ou inter-régionales}

\section{La pluviométrie}

14 Plusieurs études confirment le rôle de facteur déclencheur des variations pluviométriques sur la mobilité (Mounkaïla, 2002 ; Henry et al., 2004b). Barrios et al., (2006) à travers une approche économétrique qui croise des données de plusieurs pays depuis 1960, ont montré que les variations pluviométriques ont été un élément déterminant dans la migration des milieux ruraux vers les milieux urbains en Afrique subsaharienne. Dans un autre registre, Grolle (2015) fait une analyse historique de trois famines $^{3}$ et des migrations qui en ont découlé entre 1953 et 1985 dans le Sahel. L'analyse montre que les famines ne sont pas nécessairement le produit direct des sécheresses (Sen, 1981) mais plutôt la conséquence d'une mauvaise répartition pluviométrique durant la croissance des cultures vivrières et des choix politiques. La distribution des précipitations contribue donc aussi à initier et à favoriser la migration.

15 Comme réponse au déficit pluviométrique, différentes stratégies sont possibles, dont la migration. Pour étudier cet aspect, Rademacher-Schulz et al. (2013) examinent les relations entre les variations pluviométriques, la sécurité alimentaire et la migration dans les zones rurales au Nord du Ghana dans quatre communautés du district de Nasowli. La recherche, qui utilise une méthode mixte, a mis en avant un glissement temporel de la migration. En effet, pendant l'année 2011, il semble que les individus ont migré durant la saison des pluies qui correspond à une période de pénurie alimentaire car il n'y a aucune récolte. Cet article met en avant qu'une analyse temporelle détaillée des migrations est primordiale. Nous pouvons voir ici que pour certains ménages, la migration est un moyen de gestion du risque ex ante, tandis que pour d'autres c'est une stratégie ex post pour faire face aux modifications pluviométriques. Il ressort que les ménages mobilisant la migration ex post, augmentent souvent leur vulnérabilité et l'insécurité de leurs moyens de subsistance car la migration a lieu lors de déficit alimentaire et les travaux dans les cultures vivrières ne pourront pas être effectués. Ainsi, le facteur économique dans les migrations environnementales peut induire des inégalités et renforcer la vulnérabilité.

16 D'autres auteurs se sont intéressés au rôle de la perception de la variation des précipitations dans la décision de migrer. Zickgraf et al. (2016) ont effectué une revue de littérature des études sur la perception de la variabilité pluviométrique depuis les années 2000. Leurs résultats montrent qu'en cas de sécheresse, de climat plus sec ou plus humide, la migration n'est pas la première stratégie d'adaptation employée. Mais lorsque la situation se dégrade, l'intention de migrer augmente. Néanmoins, il n'est pas certain que les individus se déplacent car cela demande passablement de ressources financières, humaines et sociales. Par exemple à Cotonou, qui connaît une forte érosion du littoral, Zickgraf et al. (2016) ont montré que les personnes qui partent disposent de ressources suffisantes en termes de capitaux économiques et sociaux. Dans le cas contraire, les individus peuvent être dans la situation de «trapped population».

\section{Les températures}

17 Le réchauffement climatique influe sur l'augmentation globale des températures et intensifie les migrations internes aux pays principalement via la production agricole. C'est ce que Dillon et al. (2011) mettent en avant en mobilisant et en croisant des 
données statistiques d'une enquête de suivi de déplacement, d'une enquête sur la composition des ménages et de données sur les températures. Les auteurs montrent que le changement climatique a augmenté l'exode rural au Nigéria et que ce sont surtout les hommes qui migrent après des événements de dérèglement des températures.

\section{Le couvert végétal}

Une seule étude parmi celles considérées dans cet article s'intéresse au lien entre la dynamique du couvert végétal et la mobilité humaine (Van der Geest et al., 2010). Cette recherche mobilise une approche "push-pull » à travers une méthode statistique qui croise la destination de cent dix migrants au Ghana et le "Normalized Difference Vegetation Index " (NDVI). Les auteurs arrivent à la conclusion que la migration NordSud est liée à un meilleur couvert végétal. L'accès aux ressources naturelles semble prépondérant dans le choix de la destination. En effet, les populations rurales ont tendance à migrer vers des régions moins peuplées car l'accessibilité des terres cultivables en termes de prix est plus abordable.

Nous voyons ici que les déficits pluviométriques, la distribution des précipitations, l'augmentation des températures et l'évolution du couvert végétal ont un impact confirmé sur les migrations internes ou interrégionales et peuvent jouer un rôle de facteur déclencheur et/ou orienter les migrants vers certaines destinations privilégiées. A cet égard, on peut citer les zones urbaines comme particulièrement attractives notamment dans des situations de déficit pluviométrique et lorsque les températures augmentent. A contrario, il semblerait que la distribution et la dynamique du couvert végétal pousseraient les individus vers une migration rurale-rurale en direction de zones moins peuplées ${ }^{4}$.

\section{Les migrations internationales}

De manière contre-intuitive, certaines des premières études à établir un lien entre environnement et migration internationale en Afrique de l'Ouest révèlent des effets inhibiteurs ou réducteurs. A ce propos, on peut citer Henry (2004b) comme une auteure pionnière qui a amené l'idée que les changements environnementaux peuvent jouer un rôle de frein à la migration. Récemment, deux études ont confirmé ce phénomène. La première de Nawrotzki et al. (2016) étudie les relations entre des contextes d'insécurité alimentaire induite par les variations de températures et de pluviométrie entre 1960 et 2010 et les migrations au Burkina-Faso ainsi qu'au Sénégal en mobilisant la base de données Terrapop. A travers une analyse statistique, les auteurs concluent que les vagues de chaleur sont en lien avec une diminution des migrations internationales au Burkina-Faso, tandis qu'une augmentation pluviométrique accroît les migrations internationales au Sénégal. La deuxième étude se base toujours sur les données de Terrapop mais affine la méthodologie par l'intégration de données représentatives de recensements et de données mensuelles de températures et de précipitations sur une période de quarante-six années. Nawrotzki et Bakhtsiyarava (2017) questionnent de nouveau la relation entre le climat et les migrations au Burkina-Faso et au Sénégal. Cette étude examine si les mauvaises conditions climatiques agissent comme un conducteur ou un inhibiteur de la migration en mobilisant le cadre théorique de l'« agricultural pathway» qui présume que les changements climatiques auront des plus 
grands effets sur la migration dans les régions qui sont fortement dépendantes de l'agriculture. Cette étude arrive aux mêmes résultats généraux que la précédente. Les précipitations abondantes sont positivement associées avec des départs internationaux au Sénégal tandis que les grosses vagues de chaleur diminuent la probabilité de migration internationale au Burkina-Faso. Ces résultats suggèrent donc que la migration internationale peut augmenter lorsque les conditions climatiques sont favorables et qu'elle peut diminuer pendant des périodes de stress environnemental. Ceci sous-entend que les variations en ressources disponibles sont primordiales pour financer un déplacement international principalement pour les régions vivant de l'agriculture.

21 Une autre étude (Naudé, 2008) souligne cependant que les aléas environnementaux peuvent tout de même aussi favoriser indirectement les migrations internationales. L'auteur compare quarante-cinq pays sur une période allant de 1965 à 2005. Outre les raisons économiques et conflictuelles, l'environnement serait le plus important et le plus vieux déterminant de déplacement de personnes et cela à travers trois canaux : 1) la raréfaction de l'eau et des terres arables; 2) les conflits pour les ressources naturelles; 3) les catastrophes naturelles. Il est à noter que les changements climatiques participent à exacerber ces trois facteurs. L'étude conclut qu'il y a une interrelation complexe entre l'économie, les conflits et les conditions environnementales et qu'il est donc difficile de mettre en exergue un déterminant plus qu'un autre. Néanmoins, l'environnement influe sur les conflits et les opportunités de travail, ce qui affecte négativement la croissance économique.

La famille d'études qui considère l'environnement comme ayant un impact confirmé sur les migrations s'intéresse logiquement aux destinations. Il ressort donc qu'à une échelle régionale, les déficits pluviométriques ainsi que les augmentations des températures accentuent le phénomène d'exode rural tandis que les dynamiques négatives du couvert végétal poussent les individus à trouver des destinations peu peuplées hors des centres urbains.

23 En conclusion, nous pouvons observer que les changements environnementaux n'augmentent pas nécessairement les migrations internationales mais ont plutôt tendance à les freiner surtout dans les régions qui sont fortement dépendantes des ressources naturelles. Néanmoins, l'environnement peut influer sur d'autres facteurs, qui eux au contraire, peuvent favoriser la migration internationale. Les études présentées au chapitre suivant tentent précisément de rendre compte de cette complexité.

\section{L'environnement a un impact modéré sur les migrations}

De nombreux auteurs nuancent l'impact fort et direct de l'environnement sur les migrations (Swift, 1977 ; Sow et al., 2014 ; Codjoe et al., 2017) et suggèrent d'élargir le champ d'analyse à des facteurs sociaux, culturels et démographiques (Henry et al., 2002 ; Doevenspeck, 2011; Abu et al., 2014; Hummel, 2015; Romankiewicz, Doevenspeck, 2015; Liehr et al., 2016). Alors que les études qui considèrent que l'environnement a un impact confirmé sur les migrations ont une échelle d'analyse a un niveau communautaire ou national, plusieurs recherches suggèrent de s'intéresser 
aux caractéristiques socio-culturelles des individus pour comprendre les migrations dans un contexte de changement environnemental (De Haan et al., 2002; Henry et al. 2004a).

Plusieurs résultats laissent entendre qu'il est difficile voire impossible d'isoler le facteur environnemental des autres facteurs dans la décision de migrer. C'est le cas par exemple de ceux de Doevenspeck (2011), qui soutient qu'il est analytiquement impossible de prouver qu'un mouvement migratoire a une cause purement environnementale hors des désastres naturels soudains. Pour soutenir ce propos, l'auteur se base sur une approche combinée avec des méthodes quantitatives et qualitatives pour étudier la migration en milieu rural du Nord-Est du Bénin vers le centre du pays. L'auteur arrive à la conclusion que les facteurs agissant sur la décision de migrer se situent à différents niveaux et interagissent à travers le temps. Par conséquent, la migration environnementale est multi-causale et fait partie de la configuration socioculturelle complexe à un moment donné. L'étude met donc elle aussi en avant le rôle important des caractéristiques socioculturelles dans les phénomènes migratoires. À un autre niveau d'analyse, Dreier et Sow (2015) relèvent l'impact des facteurs culturels sur les pratiques migratoires. Les auteurs analysent d'une manière critique le concept de migration environnementale à travers une étude de cas conduite au Nord-Ouest du Bénin sur les motifs migratoires vers le Nigéria des membres du groupe ethnique des Bialabas. Cette recherche qualitative fut menée auprès d'agriculteurs, de membres de gouvernements locaux et des ONG actives dans la région de recherche. Les auteurs nuancent tout particulièrement la relation directe entre conditions environnementales et migration. En effet, l'étude montre qu'il est difficile de faire une distinction claire entre la dégradation environnementale et les conséquences économiques comme motivation première dans la décision de migrer. Pour les Bialabas, c'est un mélange des conditions démographiques, économiques, environnementales et sociales qui motivent les individus à migrer. Finalement, Bleibaum (2009) identifie les raisons qui poussent les habitants des régions rurales du Sénégal à migrer. Cette étude fut conduite dans le cadre du projet EACH-FOR à Dakar, dans la région de Kaolack, à Saint-Louis et dans les communautés rurales de Ndienne Lagana, Dya et Ross Mbethio et se base sur des interviews d'experts, de fonctionnaires, de chercheurs, d'universitaires, d'ONG, d'organisations internationales ainsi que sur des entretiens directifs et semi-directifs et des conversations informelles auprès d'habitants de milieux ruraux. L'auteur souligne que les récoltes ont drastiquement baissé ces trente dernières années. A cela, on peut rajouter la dégradation du sol due à l'érosion éolienne et à la salinisation, le manque d'eau à travers la réduction pluviométrique et la salinisation des eaux douces, la déforestation et la pratique de la monoculture. Si la dégradation de l'environnement joue un rôle indéniable dans la décision de migrer des individus, elle n'est pas le seul facteur à prendre en compte. De fait, les politiques publiques agricoles, les conditions économiques ainsi que des changements dans le modèle culturel sont autant de facteurs pouvant expliquer la migration. Néanmoins, il est souvent difficile de distinguer les effets des politiques publiques agricoles et les effets de la dégradation environnementale sur la décision de migrer. Récemment, Codjoe et al. (2017) ont étudié les submersions marines ${ }^{5}$ dans trois communautés du Ghana. En effectuant une enquête auprès de trois cent cinquante ménages et en mobilisant le concept d'intention de migrer, les auteurs mettent en avant qu'il n'y a pas de relation directe et linéaire entre les submersions marines et la migration. Ainsi, d'autres facteurs au niveau communautaire doivent être pris en 
compte pour comprendre l'intention de migrer des ménages tels que la culture, le niveau de développement ou l'histoire migratoire de la communauté.

Tout en nuançant l'impact de l'environnement sur les migrations, deux études proposent de réduire l'échelle d'analyse au ménage et à l'individu. De Haan et al. (2002) ont réalisé une étude dans deux villages maliens basée sur le cadre théorique du " sustainable livelihoods » et ont ainsi placé l'analyse au niveau des ménages. Ce cadre théorique permet d'intégrer en même temps un point de vue économique et anthropologique en faisant converger l'attention sur les structures institutionnelles en termes de réseaux et de gestion du ménage. Cette recherche montre que l'insécurité environnementale peut certes être un "push» facteur mais que les conditions économiques des pays voisins peuvent être considérées comme un «pull» facteur. Les auteurs nuancent par ailleurs le caractère déterministe des conditions environnementales et économiques en démontrant que les normes sociales et culturelles participent à établir qui va migrer, à quel moment et qui va en retirer les bénéfices. Une autre étude désormais classique a démontré le caractère individuel, hétérogène et non-linéaire des pratiques migratoires environnementales. Henry et al. (2004b) ont étudié l'impact des conditions pluviométriques sur les premières décisions migratoires des individus en milieu rural au Burkina-Faso. Les auteurs ont combiné des données longitudinales provenant d'une enquête nationale sur la migration avec une enquête rétrospective communautaire ainsi qu'avec des données pluviométriques entre 1960 et 1998. Cette étude relativise la relation « cause-effet » de la migration climatique et soulève l'importance d'autres facteurs tels que le niveau d'éducation ou le groupe ethnique. Ces deux études ont donc ouvert les portes à des recherches portées sur l'individu et suggèrent la prise en compte des caractéristiques socio-culturelles dans le comportement migratoire.

\section{Les études qui tentent de mesurer différents facteurs}

Comme nous venons de l'exposer, il est largement admis qu'il est difficile d'appréhender des migrations comme purement environnementales. Certains auteurs qui reconnaissent l'impact modéré de l'environnement tentent cependant de pondérer l'effet des différents facteurs dans un contexte de multicausalité.

\section{Un phénomène historique}

De nombreux auteurs soulignent qu'en Afrique sub-saharienne, la migration a toujours été utilisée comme un moyen de sécuriser des moyens de subsistance. L'emploi de la migration circulaire et temporaire vers les zones côtières, les zones « urbaines » et au sein des zones rurales préexistait à la colonisation française et s'est accentué avec la capitalisation de la société traditionnelle (Van der Land et al., 2018). La mobilité est donc ancrée dans les structures culturelles de l'Afrique de l'Ouest (Mortimore, 1989; Mounkaïla, 2002 ; Hummel, 2015).

Déjà en 1977, Swift (1977) montrait à travers une approche historique et avec l'utilisation d'une description de l'économie des populations pastorales dans le Sahel, que les famines ont un aspect structurel en raison des non-agissements au niveau de l'implémentation de politiques publiques, mais aussi de l'héritage de la conquête coloniale et des vestiges de son administration. Cet article nuance donc le poids de la sécheresse comme cause directe de la famine et des migrations et fait intervenir le 
facteur politique ainsi que la variable structurelle dans la relation entre changements climatiques et mobilité. Déjà lors des premières études réalisées sur les liens entre migration et dégradations environnementales le discours était nuancé quant à la relation entre les conditions environnementales et les migrations. Swift met donc en exergue l'influence structurelle des politiques publiques sur l'environnement et sur les conditions de vie des populations pastorales. Quant à Van der Geest $(2009,2011)$, il s'intéresse à la migration du nord vers le sud du Ghana. Suite à des entretiens menés auprès de deux cent trois agriculteurs migrants, il ressort que les mauvaises conditions agro-écologiques au Nord poussent les fermiers à s'installer en direction du Sud où le climat est plus humide. Néanmoins, la migration ne serait pas directement liée à la dégradation environnementale mais plus à la pénurie structurelle des ressources. La migration serait donc un moyen de faire face à cette pénurie et au manque d'opportunités afin d'assurer un revenu qui sécurise un minimum de moyens de subsistance. Cette étude met en lumière que les migrations environnementales peuvent être le résultat direct des déficiences structurelles en termes de ressources naturelles disponibles.

Roquet (2008), avec une synthèse bibliographique et des enquêtes biographiques, montre qu'au Sénégal la migration n'est pas un phénomène nouveau qui s'inscrit dans un ensemble de stratégies de lutte contre la pauvreté et pour la durabilité. La conclusion de Romankiewicz et al. (2016) rejoint à quelques égards celle de Roquet (2008). Les auteurs s'intéressent à la continuité de la pratique de la migration dans le village de N'Guith au Sénégal qui a une longue pratique migratoire. Il est démontré que le début et la continuité de la mobilité des habitants du village sont structurés par le contexte sociohistorique de la communauté. Le développement du village et la diversité en cultures maraîchères sont donc supportés par les migrants. Toujours à une échelle locale, Delaunay et al. (2016) mobilisent des enquêtes qualitatives qui sont mises en parallèle avec des données quantitatives longitudinales d'un observatoire de la population de Niakhar au Sénégal. Cette étude de cas dans la commune de Toucar met en avant que les changements écologiques, économiques et sociaux ont transformé et diffusé la pratique de la migration temporaire. Ainsi, on assiste non pas à une migration de rupture mais à une migration qui permet de structurer la résilience du village.

Dans le même registre, De Bruijn et Van Dijk (2003) se penchent sur les migrants ruraux appartenant à l'ethnie des Fulbes au centre et au Sud du Mali. Les résultats mettent en avant que la migration a des ancrages culturels en termes de réseaux mais surtout en termes d'appartenance ethnique. Les dégradations environnementales ont modifié les pratiques de mobilité traditionnelles dans le temps, l'espace et au sein même des structures culturelles. Dans ce sens, Afifi $(2009 ; 2011)$ explique que même si la migration saisonnière fait partie intégrante de la culture au Niger, la migration permanente au sein du pays et vers d'autres Etats africains est devenue commune surtout pour les individus qui dépendent fortement des ressources naturelles en raison des nombreux problèmes environnementaux tels que la désertification, la dégradation des sols, les sécheresses. Les caractéristiques de la mobilité en Afrique subsaharienne ont également évolué en miroir des changements sociétaux. Au Mali et au Sénégal, Romankiewicz et Doevenspeck (2015) montrent que la migration saisonnière et temporaire a été largement utilisée comme stratégie d'adaptation aux conditions climatiques avant l'émergence du débat international sur le changement climatique mais peut également être considérée comme une stratégie d'adaptation aux changements sociaux, économiques et culturels. Les auteurs arrivent à la conclusion 
que les caractéristiques de la migration contemporaine ont changé sous l'influence de facteurs sociaux. À Saint-Louis au Sénégal, Zickgraf et al. (2016) ont montré que la montée du niveau de la mer, l'érosion côtière et la baisse en ressources halieutiques ont modifié les comportements migratoires. En effet, si les pêcheurs du quartier de Guet Ndar ont toujours mis en place des stratégies de migration circulaire afin de suivre les bancs de poissons, la dégradation de l'environnement pousse désormais principalement les jeunes hommes à partir plus loin, plus longtemps et souvent de manière permanente.

L'insécurité alimentaire ou les variations pluviométriques ont également des impacts sur les structures familiales et les pratiques migratoires habituelles. C'est ce que Mounkaïla (2002) met en avant en s'intéressant aux relations existantes entre l'insécurité alimentaire comme résultante de l'incertitude pluviométrique et à la migration comme stratégie de gestion de cette situation précaire au Niger. Il définit les caractéristiques de cette migration particulière et la manière dont elle s'inscrit dans l'espace, sachant que la mobilité spatiale est une pratique traditionnelle. Les communautés ont ainsi développé deux logiques de mobilité. La première vise à maintenir l'unité familiale au sein du territoire local, en cherchant des ressources nécessaires à la survie à l'extérieur du territoire. Cette stratégie rend les unités familiales extrêmement dépendantes de la migration circulaire et augmente le phénomène migratoire. La deuxième logique consiste à abandonner le territoire en transférant les unités familiales et les activités économiques dans un autre lieu. En définitive, les diverses pratiques migratoires révèlent les choix opérés par les familles, qui ne sont pas sans impact sur les dynamiques affectives. En effet, certains choix tendent vers la diversification de revenus, tandis que d'autres tendent vers l'éclatement et l'effritement des structures familiales par le départ définitif de certains de leurs membres. Cette situation pose la question du devenir de ces communautés et de leurs territoires et montre que les mouvements migratoires peuvent également modifier la démographie d'un territoire.

\section{L'impact de la migration}

La migration peut avoir des impacts négatifs sur l'environnement. Un type de cercle vicieux peut alors se mettre en place. La dégradation environnementale encourage des migrations, qui elles-mêmes participent à dégrader l'environnement dans les lieux de destination.

Le projet EACH-FOR (Afifi, 2009) met en lumière le mécanisme économique à travers lequel les facteurs environnementaux ont un impact sur la migration au Niger. Pour ce faire, Afifi a mené des interviews et des questionnaires auprès d'experts, auprès de migrants et auprès de personnes touchées par la dégradation environnementale mais qui ont décidé de ne pas migrer. La conclusion de cette recherche observe que la dégradation environnementale a un impact multidimensionnel sur la migration au Niger. Un cercle vicieux qui lie migration et dégradation environnementale se met en place et ces deux phénomènes se renforcent mutuellement. Dans le cas du Niger, il est donc clair que les perturbations environnementales ont changé les habitudes migratoires et ont induit des migrations économiques qui elles-mêmes participent à dégrader l'environnement. Au Sénégal, Failler et Binet (2010) se sont intéressés aux pêcheurs migrants. En utilisant une approche historique des activités des pêcheurs depuis les sécheresses des années septante et de l'évolution du cadre formel de la 
pêche, ils montrent que les premiers pêcheurs migrants étaient eux-mêmes des déplacés climatiques qui ont su tirer profit des eaux poissonneuses du Sénégal. Néanmoins, les phénomènes migratoires se sont intensifiés poussant les pêcheurs à étendre leurs activités dans le temps et l'espace en franchissant les frontières des eaux territoriales. Ainsi, sans un cadre institutionnel fort, la raréfaction des ressources halieutiques est inévitable. Cette recherche suggère que la migration environnementale elle-même peut avoir des impacts néfastes sur l'environnement et ainsi augmenter le phénomène migratoire.

\section{L'environnement et les facteurs sociodémographiques} caractéristiques sociodémographiques des individus dans leur décision de migrer. Les chercheurs évaluent l'importance du rôle de ces facteurs dans la migration interprovinciale au Burkina-Faso. Les résultats émergeant de l'utilisation d'un modèle statistique qui croise des données sociodémographiques émanant du recensement de 1985 et des données environnementales, montrent que les caractéristiques sociodémographiques ont plus d'influence sur les motifs à la migration interprovinciale que la dégradation environnementale. En lien avec la conclusion de Henry et al. (2002), Abu et al. (2014) utilisent les données du "Climate Change Collective Learning " et de l' "Observatory Network Ghana Project » dans une régression logistique pour examiner les intentions migratoires des ménages face à des stress environnementaux. Leurs résultats montrent que les facteurs entrant en jeu dans la décision de migrer ont plusieurs dimensions. En effet, même dans les zones géographiques où il y a des stress environnementaux récurrents, l'environnement n'est pas le premier motif dans la décision de migrer. Les auteurs avancent donc que les facteurs sociodémographiques sont de meilleurs éléments de prédictions migratoires que les événements climatiques eux-mêmes. Au Niger, Rain (1999) étudie les liens entre les sécheresses et les famines depuis les années soixante. Avec des méthodes qualitatives, l'auteur souligne l'importance des réseaux dans les migrations circulaires lors de crise environnementale et de considérer le migrant en tant qu'agent qui fait des choix individuels. Dans un autre registre, Findley (1995) étudie la migration rurale au Mali lors de la sécheresse de 1983-1985. En décrivant les caractéristiques des personnes ayant migré, l'auteure met en lumière la féminisation de la migration et son aspect circulaire et court dans le temps. Cette recherche souligne la dimension de genre de la migration et ses pratiques temporelles hétérogènes.

Si l'on resserre l'analyse à niveau très micro, l'éducation ainsi que l'appartenance religieuse influencent aussi les pratiques migratoires. Il est à noter que ces facteurs ont été que très peu étudiés et qu'il n'y a quasiment pas de travaux sur la thématique de la religion.

Van der Land et Hummel (2013) s'intéressent au rôle de l'éducation formelle dans la relation entre changements climatiques et migration au Mali et au Sénégal. En combinant des méthodes quantitatives et qualitatives, les auteures mettent en avant 
que l'éducation a un rôle important dans la réduction de la vulnérabilité car les personnes avec un capital éducationnel élevé sont moins vulnérables aux changements climatiques car moins dépendantes de l'agriculture de subsistance. Néanmoins, leurs résultats montrent que le niveau d'éducation n'a pas d'influence sur l'expérience migratoire en tant que telle mais sur les motivations. Par conséquent, la migration apparaît comme une stratégie de réduction des risques pour les individus n'ayant pas suivi une éducation formelle ou alors ayant un niveau primaire. Alors que pour les individus mieux éduqués, la migration est une stratégie pour améliorer le bagage éducationnel. D'autres auteurs se sont intéressés à l'impact de l'éducation sur la migration. Ajaero et al. (2016) examinent ainsi l'impact des inondations de 2012 sur les ménages migrants dans les communautés rurales dans le Sud-Est du Nigéria. Cette recherche basée sur des méthodes mixtes s'intéresse aux moyens de subsistance. Il en ressort que les communautés les plus vulnérables aux inondations doivent mettre en place des stratégies d'adaptation dont la migration. Pour les ménages qui ont pris cette option, la migration conduit à une perte de propriété foncière, à une réduction des moyens de subsistance et à une diminution de la qualité de vie en général. Finalement, cette recherche montre l'importance de l'éducation et des revenus provenant d'activités non-agricoles dans la sécurisation des moyens de subsistance après la migration.

Pour aborder le facteur de la religion, Purokayo et Gabdo (2013) étudient onze quartiers à Jumeta-Yola au Nigéria. Cette étude qualitative montre que la décision de migrer dépend fortement de la perception de la sécurité et des facteurs ethnico-religieux. En effet, l'insécurité causée par les actions de groupes fondamentalistes religieux a poussé des individus à quitter leur habitat d'origine pour aller s'établir dans des zones où l'environnement est moins favorable mais où la perception de sécurité liée aux facteurs ethnico-religieux est plus forte.

\section{Encadré 2}

\section{A chaque aléa sa migration}

La migration en Afrique de l'Ouest n'est pas homogène, notamment en raison de la grande diversité des impacts du changement climatique et des multiples dégradations environnementales. C'est ce que Neumann et al. (2015) relèvent. Il semblerait que la dégradation du sol a tendance à provoquer plus de phénomènes migratoires que l'accès aux ressources en eau. Le type de migration dans le temps et l'espace dépend également du type de stress environnemental. Henry et al. (2004a) mettent en avant qu'au Burkina-Faso les conditions agro-climatiques dans le long ou le court terme vont produire des migrations permanentes ou temporaires. Toujours au Burkina-Faso et dans une autre étude, Henry et al. (2004b) soulignent que la décision de migrer est plus influencée par des processus lents de dégradation environnementale que par des événements soudains.

41 Nous pouvons donc voir que des données sociodémographiques peuvent être de bons éléments de prédictions de l'intention de migrer. À un niveau macro et meso, on peut citer le taux de chômage, le taux d'alphabétisation et la taille des ménages. À niveau plus micro, le genre, le niveau d'éducation, l'âge et l'histoire migratoire semblent également être de bons indicateurs. Finalement, nous relevons qu'aucune étude de cas ne fait référence à la pression démographique. 


\section{Les approches globales} environnementaux. L'auteure examine l'interaction entre le changement climatique, la dégradation des sols et la migration dans la région de Bandiagara au Mali et dans la région de Linguère au Sénégal. Pour examiner cette interaction complexe, il est primordial de mettre en relation les multiples facteurs qui influencent la migration. Pour ce faire, ces relations sont conceptualisées comme un "social-ecological system ». L'analyse est basée sur un projet interdisciplinaire nommé « migration, climate change and environment in the Sahel » (MICLE) qui prend la dégradation des sols comme un exemple de "slow on-set event » et la met en relation avec les mouvements migratoires. Ce projet s'est basé sur une enquête auprès de neuf cent cinq individus. Ce concept suggère que les stratégies et les actions mises en place par un individu, un groupe ou une société pour faire face aux changements environnementaux dépendent des relations sociétales avec la nature. Ces relations spécifiques qui émergent des variables culturelles et historiques, modèlent les relations de l'Homme à son environnement. Ainsi, la dégradation du sol n'est pas seulement les résultats de dynamiques biophysiques mais bien des décisions et des actions humaines. Ces actions et ces décisions sont elles-mêmes dépendantes et influencées par les conditions sociales, culturelles, politiques et économiques qui sont à leur tour influencées par les conditions environnementales. Par conséquent, les raisons et les motivations de la migration ne peuvent pas être simplement divisées entre les motifs écologiques, sociétaux ou simplement expliqué par la théorie «push-pull » mais doivent être compris comme un tout. Les résultats de cette étude montrent que la mobilité est une caractéristique de l'Afrique de l'Ouest et qu'elle doit être appréhendée dans le contexte des dynamiques régionales.

Néanmoins, l'utilisation de la migration comme stratégie pour faire face aux dégradations environnementales reste souvent le seul moyen pour les populations les plus précaires de sécuriser leurs moyens de subsistances. Liehr et al. (2016) ont continué le travail initié par Hummel (2015) et mobilisent également le cadre conceptuel du " social-ecological system " et les données du projet MICLE. Les auteurs arrivent à la conclusion que la migration est une réponse sociétale dans un contexte de changements climatiques et de dégradations environnementales. Pour ce faire, les auteurs utilisent l'approche de modélisation intégrative du "Bayesian Belief Network » (BBN) dans le but d'obtenir une meilleure compréhension des interactions entre les conditions environnementales et les conditions socioéconomiques. Pour cette approche, les variables environnementales et socioéconomiques interagissent entre elles et impactent les motifs migratoires qui, à leur tour, influencent la décision de migrer et les spécificités temporelles et spatiales de la migration. La question de la 
migration comme stratégie d'adaptation est donc abordée et discutée. Suite à leurs enquêtes qualitatives et quantitatives, les auteurs concluent que la migration peut être considérée comme une stratégie d'adaptation pour les ménages et un moyen pour diversifier leurs revenus. Puis, il s'agit de savoir qui a les moyens de mettre une telle stratégie en place. En effet, il faut prendre en compte que les conditions économiques et sociales peuvent restreindre l'adaptation. Ainsi, une différence temporelle et spatiale est faite entre les stratégies d'adaptation et les stratégies pour faire face aux dégradations environnementales. Selon Liehr et al. (2016), les stratégies d'adaptation s'étendent dans le temps et dans la distance, tandis que les " coping " stratégies peuvent être vue comme une réponse immédiate à des conditions agro-écologiques difficiles.

\section{Conclusion}

Cette revue de littérature a fait le point sur l'état de la recherche sur les migrations environnementales en Afrique de l'Ouest. Ci-dessous nous résumons dix enseignements et acquis de la recherche. Nous avons classé les différents énoncés ci-dessous suivant le système de classement de l'IPCC en estimant le degré d' "agreement » (consensus entre les chercheurs) et d' «evidence » (nombre et ampleur des études de cas) (IPCC 2010).

1. La mobilité est ancrée dans les structures culturelles et coutumières de l'Afrique de l'Ouest (Mortimore, 1989 ; Mounkailla, 2002 ; De Bruijn, Van Dijk, 2003 ; Roquet, 2008 ; Afifi, 2009 ; Afifi, 2011; Morrissey, 2014; Hummel, 2015; Romankiewicz et Doevenspeck, 2015 ; Romankiewicz et al., 2016 ; Delaunay et al., 2016; Zickgraf et al., 2016). High agreement/ Robust evidence

2. Les déficits pluviométriques ainsi que l'augmentation des températures semblent être les deux aléas qui ont le plus d'influence sur les migrations internes ou inter-régionales. (Mounkailla, 2002 ; Henry et al., 2004b ; Dillon et al., 2011 ; Rademacher-Schulz et al., 2013 ; Grolle, 2015 ; Barrios et al., 2016) High agreement/Robust evidence

3. Le Sahel ainsi que plus largement l'Afrique de l'Ouest sont des zones qui sont et seront fortement impactées par les changements environnementaux (IPCC, 2014; Müller et al., 2014 ; Neumann et al., 2015). Ces zones sont à considérer comme des « hot-spots » sur le plan international. High agreement/Robust evidence

4. Les principales zones de départ sont les régions rurales (Mortimore, 1989 ; Findley, 1995 ; Mounkaïla, 2002 ; Henry et al., 2004a ; Van der Geest, 2010 ; Dillon et al., 2011 ; Doevenspeck, 2011 ; Rademacher-Schulz et al., 2013; Hummel, 2015 ; Ajaero et al., 2016) et les centres urbains sont les principales destinations (Pedersen, 1995; Barrios et al., 2006 ; Dillon et al., 2011). High agreement/Robust evidence

5. Le facteur environnemental ne suffit pas pour comprendre et expliquer des migrations dans un contexte de stress climatique. Il faut ouvrir l'analyse à d'autres facteurs tels que des facteurs sociaux, des facteurs culturels, des facteurs démographiques, des facteurs politiques et des facteurs économiques (Swift, 1977 ; Henry et al., 2002 ; Abu et al., 2014 ; Sow et al., 2014 ; Hummel, 2015 ; Romankiewcz et Doevenspeck, 2015 ; Liehr et al., 2016 ; Romankiewicz et al., 2016 ; Codjoe et al., 2017). High agreement/Robust evidence

6. Les changements environnementaux peuvent modifier des habitudes migratoires préexistantes (Mounkaïla, 2002 ; de Bruijn et Van Dijk, 2003 ; Afifi, 2009 ; Rademacher-Schulz et al., 2013 ; Zickgraf et al., 2016). High agreement/Medium evidence

7. L'environnement a un impact confirmé concernant les migrations sur de courtes distances et pour de courtes périodes (Mortimore, 1989; Findley, 1995; Mounkaïla, 2002; Van der Geest et al., 2010 ; Hummel, 2015). High agreement/Medium evidence 
8. L'approche monocausale en termes de «push-pull » semble dépassée. Les recherches doivent s'ouvrir à l'interaction entre différents facteurs dans les causes migratoires. En raison des caractéristiques hétérogènes et particulières d'une migration, il est analytiquement difficile voire impossible de prouver qu'elle soit uniquement de cause environnementale (De Haan et al., 2002 ; Henry et al., 2004a ; Bleibaum, 2009 ; Doevenspeck, 2011 ; Dreier et Sow, 2015). High agreement/Medium evidence

9. Les villes africaines tendent actuellement à attirer les migrations. En raison de leur concentration sur le littoral, ces dernières risquent cependant aussi devenir des zones de départ en raison de l'augmentation tendancielle du niveau des océans et des conséquences qui en découlent (Doussou et Glehouenou-Doussou, 2007; Zickgraf et al., 2016). Medium agreement/Limited evidence

10. Les dégradations environnementales peuvent freiner les possibilités de migration internationale car cette dernière demande beaucoup de ressources (Henry, 2004b ; Naudé, 2008 ; Nawrotzki et al., 2016 ; Nawrotzki et Bakhtsiyarava, 2017). Ces résultats nuancent les prédictions d'auteurs alarmistes qui présageaient des flux internationaux massifs de population (Myers, 1997). Medium agreement/Limited evidence

La relation entre les changements environnementaux et la migration en Afrique de l'Ouest comporte quelques spécificités. La mobilité fait ainsi plus qu'ailleurs partie intégrante de la culture de la sous-région. Néanmoins, la dégradation environnementale a modifié les habitudes migratoires. A cet égard, l'environnement semble avoir un impact fort sur les migrations de courtes distances. Cela prend toute son ampleur lorsque l'on sait que la majorité des migrations, dans un contexte de changements environnementaux ou climatiques, sont inter ou intrarégionales. $\mathrm{Au}$ niveau des aléas environnementaux, ce sont les variations pluviométriques et l'augmentation des températures qui influencent le plus les migrations. Toutefois, les dégradations environnementales peuvent avoir un rôle d'inhibiteur des migrations surtout au niveau international. Finalement, les régions rurales sont les principales zones de départ. Les centres urbains qui se situent principalement sur le littoral sont particulièrement attractifs mais ces derniers pourraient eux-mêmes être des zones de départ en raison de la montée tendancielle des océans.

$\mathrm{Au}$ regard de l'état des lieux méthodologique ${ }^{6}$ des quarante-trois études de cas présentées, nous pouvons mettre en avant des lacunes des travaux existants et des directions de recherches futures. Si l'on s'intéresse à l'échelle d'analyse, il apparaît que le niveau national est surreprésenté comparé aux niveaux meso et micro. Il conviendrait donc de resserrer l'analyse aux contextes sociaux, économiques et politiques des communautés et aux stratégies des ménages ainsi qu'aux choix des individus. Concernant les méthodes de collecte de données, il ressort clairement que les techniques quantitatives et qualitatives ont été préférées aux méthodes mixtes ou historiques. L'utilisation accrue de ces dernières semble donc pertinente. On constate aussi que les études de cas se sont principalement basées sur des aléas environnementaux avérés et objectifs. Il serait donc intéressant d'orienter les études vers la perception subjective des changements environnementaux par les individus. Enfin, ce sont principalement les aléas environnementaux progressifs (slow on-set event) qui ont le plus été pris en compte et la recherche sur les événements ponctuels à caractère exceptionnels devrait être intensifiée. En effet, il est reconnu que les impacts du changement climatique vont accentuer la fréquence et/ou l'intensité de ces derniers. 

titre que d'autres facteurs. Ainsi, d'un point de vue théorique, il semble que l'approche monocausale du "push-pull» soit dépassée et trop simpliste. Par conséquent, la recherche doit s'orienter vers des études plus compréhensives et multivariées pour examiner les migrations dans un contexte de dégradation environnementale. Parmi les thématiques demandant à être développées nous avons relevé : les causes politiques et structurelles, les contextes sociodémographiques, et finalement les aspects liés au capital humain (éducation), à la religion et aux représentations culturelles et coutumières. Il est à souligner que ces deux dernières thématiques ont été particulièrement peu explorées et qu'elles mériteraient une multiplication de cas d'étude. Enfin, les effets de seuils et les boucles de rétroaction (cercles vicieux) entre migration et environnements devraient aussi être étudiés de manière plus intensive.

Compte tenu des natures diverses des aléas environnementaux et de l'hétérogénéité des réponses qui ressortent de notre synthèse, il peut sembler illusoire de vouloir élaborer une théorie générale pour expliquer et prédire les migrations environnementales en Afrique de l'Ouest. En identifiant dix régularités remarquables dans les études existantes nous avons néanmoins pu montrer qu'une certaine généralisation était possible. L'intégration des études futures dans un cadre conceptuel commun reste souhaitable. L'espoir de la synthèse qui précède est d'en faciliter l'émergence.

\section{BIBLIOGRAPHIE}

ABU M., CODJOE S. \& SWARD J. (2014), “Climate change and internal migration intentions in the forest-savannah transition zone of Ghana", Population and Environment, 35, 4, pp. 1-24.

AFIFI T. (2009), Niger: Case Study Report for the EACH-FOR Project, United Nations University EHS.

AFIFI T. (2011), “Economic or environmental migration? The push factors in Niger», International Migration, 49, S1, pp. 95-124.

AJAERO C.K., MOZIE A. T. \& ABU I. N. (2016), “Migrating from Migratory Waters to Migration of Livelihoods", Social Indicators Research, pp. 1-15.

BARRIOS S., BERTINELLI L. \& STROBL E. (2006), “Climatic change and rural-urban migration: The case of sub-Saharan Africa”, Journal of Urban Economics, 60, 3, pp. 357-371.

BEAUCHEMIN C., BOCQUIER P. (2004), "Migration and urbanisation in Francophone West Africa: An overview of the recent empirical evidence", Urban Studies, 41, 11, pp. 2245-2272.

BLEIBAUM F. (2009), Senegal: Case Study Report for the EACH-FOR Project, United Nations University EHS.

CODJOE S., NYAMEDOR F., SWARD J.et DOVIE D. (2017), "Environmental hazard and migration intentions in a coastal area in Ghana: a case of sea flooding", Population and Environment, pp. 1-19. 
DE BRUIJN M., VAN DIJK H. (2003), “Changing population mobility in West Africa: Fulbe pastoralist in Central and South Mali", African Affairs, 102, 407, pp. 285-307.

DE HAAN A., BROCK K. \& COULIBALY N. (2002), “Migration, livelihoods and institutions: contrasting patterns of migration in Mali”, The Journal of Development Studies, 38, 5, pp. 37-58.

DELAUNAY V., ENGELI E., FRANZETTI R., GOLAY G., MOULLET A. \& SAUVAIN-DUGERDIL C. (2016), "La migration temporaire des jeunes au Sénégal. Un facteur de résilience des sociétés rurales sahéliennes?", Afrique contemporaine, 259, pp. 75-94.

DILLON A., MUELLER V. \& SALAU S. (2011), “Migratory Responses to Agricultural Risk in Northern Nigeria", American Journal of Agricultural Economics, 93, 4, pp. 1048-1061.

DOEVENSPECK M. (2011), “The Thin Line Between Choice and Flight: Environment and Migration in Rural Benin”, International Migration, 49, supplément, pp. 50-68.

DOSSOU K. L. M. R., GLEHOUENOU-DOSSOU B. (2007), “The vulnerability to climate change of Cotonou (Benin): the rise in sea level”, Environment and Urbanization, 19, 1, pp. 65-79.

DREIER V., SOW P. (2015), "Bialaba Migrants from the Northern of Benin to Nigeria, in Search of Productive Land - Insights for Living with Climate Change”, Sustainability, 7, 3, pp. 3175-3203.

FAILLER P., BINET T. (2010), "Sénégal. Les pêcheurs migrants : réfugiés climatiques et écologiques”, Hommes et migrations, 1248, mars-avril, pp. 98-111.

FINDLEY S. E. (1995), "Does drought increase migration? A study of migration from rural Mali during the 1983-85 drought", International Migration Review, 28, 3, pp. 539-553.

GROLLE J. (2015), "Historical case studies of famines and migrations in the West African Sahel and their possible relevance now and in the future", Population and Environment, pp. 1-26.

GUÉLAT J., KAENZIG R. \& PIGUET E. (2016), “Geography of research on environmental migration”, in IONESCO D., MOKHNACHEVA D. \& GEMENNE F., The Atlas of Environmental Migration, pp. 10-11.

HENRY S., BOYLE P. \& LAMBIN E. F. (2003), “Modelling inter-provincial migration in Burkina Faso: The role of socio-demographic and environmental factors", Applied Geography, 23, 2-3, pp. 115-136.

HENRY S., PICHÉ V., OUÉDRAOGO D. \& LAMBIN E. F. (2004a), “Descriptive Analysis of the Individual Migratory Pathways According to Environmental Typologies", Population and Environment, 25, 5, pp. 397-422.

HENRY S., SCHOUMAKER B. \& BEAUCHEMIN C. (2004b), "The impact of rainfall on the first outmigration: A multi-level event-history analysis in Burkina Faso", Population and Environment, 25, 5, pp. 423-60.

HUMMEL D. (2015), "Climate change, land degradation and migration in Mali and Senegal - some policy implications", Migration and Development, pp. 1-23.

IPCC (2010), Guidance note for lead authors of the IPCC fifth assesment report on consistent treatment of uncertainties, WMO/UNEP, Intergovernmental Panel on Climate Change.

IPCC (2014), Climate Change 2014 - Synthesis Report, W. UNEP, Intergovernmental Panel on Climate Change.

JONSSON G. (2010), The environmental factor in migration dynamics - a review of African case studies, Working Papers - International Migration Institute - University of Oxford I. M. Institute, University of Oxford. 
LIEHR S., DREES L. \& HUMMEL D. (2016), "Migration as Societal Response to Climate Change and Land Degradation in Mali and Senegal", in CHAM A.J., YARO J. \& HESSELBERG J. (eds.), Adaptation to Climate Change and Variability in Rural West Africa, pp. 147-169.

MORRISSEY J. (2014), "Environmental change and human migration in Sub-Saharan Africa”, in PIGUET E., LACZKO F. (eds.), People on the move in a changing climate, pp. 81-109, Springer.

MORTIMORE M. (1989), Adapting to drought: Farmers, famines, and desertification in West Africa, Cambridge University Press.

MOUNKAÏLA H. (2002), “De la migration circulaire à l'abandon du territoire local dans le Zarmaganda (Niger)”, Revue Européenne des Migrations Internationales, 18, 2, pp. 161-187.

MÜLLER C., WAHA K., BONDEAU A. \& HEINKE J. (2014), "Hotspots of climate change impacts in sub-Saharan Africa and implications for adaptation and development", Global change biology, 20, 8, pp. 2505-2517.

MYERS N. (1997), “Environmental refugees”, Population and Environment, 19, 2, pp. 167-182.

NAUDÉ W. (2008), “Conflict, disasters and no jobs: Reasons for international migration from subSaharan Africa", United Nations University WIDER Research Paper, 85.

NAWROTZKI R., BAKHTSIYARAVA M. (2017), “International climate migration: Evidence for the climate inhibitor mechanism and the agricultural pathway", Population, Space and Place, 23, pp. 4.

NAWROTZKI R. J., SCHLAK A. M. \& KUGLER T. A. (2016), “Climate, migration, and the local food security context: introducing Terra Populus", Population and Environment, 38, 2, pp. 164-184.

NEUMANN K., SIETZ D., HILDERINK H., JANSSEN P., KOK M. \& VAN DIJK H. (2015), “Environmental drivers of human migration in drylands - A spatial picture", Applied Geography, 56, pp. 116-126. PEDERSEN J. (1995), “Drought, Migration and Population Growth in the Sahel: The Case of the Malian Gourma: 1900-1991”, Population Studies, 49, pp. 111-126.

PIGUET E. (2010a), "Linking climate change, environmental degradation, and migration: a methodological overview”, Wiley Interdisciplinary Reviews: Climate Change, 1, 4, pp. 517-524.

PIGUET E., PÉCOUD A. \& DE GUCHTENEIRE P. (2010b), “La circulation des personnes. Migrations et changements climatiques", Maison d'Analyse des Processus Sociaux, Working paper $N^{\circ} 10$.

PICHÉ V., CORDELL D. (2015), Entre le mil et le franc: Un siècle de migrations circulaires en Afrique de l'Ouest. Le cas du Burkina Faso, Presses de l'Université du Québec.

PUROKAYO S.G., GABDO Y. (2013), "Implications of environment security-related factors as determinant of residential mobility in Jimeta-Yola, Nigeria", International Journal of Arts and Commerce, 2, 2, pp. 305-315.

RAIN A. (1999), Eaters of the dry season. Circular labor migration in the west African Sahel, Routledge.

RADEMACHER-SCHULZ C., SCHRAVEN B. \& MAHAMA E. (2013), "Time matters: shifting seasonal migration in Northern Ghana in response to rainfall variability and food insecurity", Climate and Development, 6, 1, pp. 46-52.

ROMANKIEWICZ C., DOEVENSPECK M. (2015), "Climate and Mobility in the West African Sahel: Conceptualising the Local Dimensions of the Environment and Migration Nexus", in GRESCHKE H., TISCHLER J. (eds.), Grounding Global Climate Change, Springer, pp. 79-100.

ROQUET D. (2008), "Partir pour mieux durer: la migration comme réponse à la sécheresse au Sénégal?”, Espace, Populations, Sociétés, 1, pp. 37-53. 
SEN A. (1981), Poverty and famines: an essay on entitlement and deprivation, Oxford University Press.

SOW P., ADAAWEN S. A. \& SCHEFFRAN J. (2014), "Migration, Social Demands and Environmental Change amongst the Frafra of Northern Ghana and the Biali in Northern Benin", Sustainability, 6, 1, pp. 375-398.

SWIFT J. (1977), "Sahelian Pastoralists: Underdevelopment, Desertification, and Famine", Annual Review of Anthropology, 6, pp. 457-478.

VAN DER GEEST K. (2008), Migration and natural resources scarcity in Ghana: Case study Report for the EACH FOR Project, United Nations University EHS.

VAN DER GEEST K. (2011), “North-south migration in Ghana: What role for the environment?”, International Migration, 49, 1, pp. 69-94.

VAN DER GEEST K., VRIELING A. \& DIETZ T. (2010), "Migration and environment in Ghana: A cross-district analysis of human mobility and vegetation dynamics", Environment \& Urbanization, 22, 1, pp. 107-123.

VAN DER LAND V., HUMMEL D. (2013), "Vulnerability and the Role of Education in Environmentally Induced Migration in Mali and Senegal”, Ecology and Society, 18, 4,

VAN DER LAND V., ROMANKIEWICZ C. \& VAN DER GEEST K. (2018), "Environmental change and migration. A review of West African case Studies”, in MCLEMAN R., GEMENNE F. (eds.), Routledge Handbook of Environmental Migration and Displacement, London, Routledge.

ZICKGRAF C., VIGIL DIAZ TELENTI S., DE LONGUEVILLE F., OZER P. \& GEMENNE F. (2016), The Impact of Vulnerability and Resilience to Environmental Changes on Mobility Patterns in West Africa, KNOMAD Working paper 14, World Bank.

\section{ANNEXES}

\section{Aperçu des études de cas prises en compte}

\begin{tabular}{|l|l|l|l|l|l|}
\hline Etudes de cas & $\begin{array}{l}\text { Echelle } \\
\text { d'analyse }\end{array}$ & Méthode & $\begin{array}{l}\text { Mesures des } \\
\text { caractéristiques } \\
\text { environnementales }\end{array}$ & $\begin{array}{l}\text { Changements } \\
\text { environnementaux }\end{array}$ & $\begin{array}{l}\text { Type de } \\
\text { migration }\end{array}$ \\
\hline $\begin{array}{l}\text { Identification } \\
\text { des zones à } \\
\text { risque }\end{array}$ & & & & \\
\hline Pedersen (1995) & Pays & Quantitatif & Faits avérés & Slow on-set & Interne \\
\hline $\begin{array}{l}\text { Doussou } \\
\text { Glehouenou- } \\
\text { Doussou (2007) }\end{array}$ & Ville & Quantitatif & Simulation & $\begin{array}{l}\text { Slow on-set et } \\
\text { Rapid on-set }\end{array}$ & Interne \\
\hline $\begin{array}{l}\text { Müller et al. } \\
\text { (2014) }\end{array}$ & Continentale & Quantitatif & Simulation & $\begin{array}{l}\text { Slow on-set et } \\
\text { Rapid on-set }\end{array}$ & Internationale \\
\hline
\end{tabular}




\begin{tabular}{|c|c|c|c|c|c|}
\hline $\begin{array}{l}\text { Neumann et al. } \\
(2015)\end{array}$ & Continentale & Quantitatif & Simulation & $\begin{array}{l}\text { Slow on-set et } \\
\text { Rapid on-set }\end{array}$ & Internationale \\
\hline $\begin{array}{l}\text { L'environnement } \\
\text { a un impact } \\
\text { confirmé }\end{array}$ & & & & & \\
\hline $\begin{array}{l}\text { Les migrations } \\
\text { internes ou } \\
\text { inter-régionales }\end{array}$ & & & & & \\
\hline La pluviométrie & & & & & \\
\hline $\begin{array}{l}\text { Barrios et al. } \\
(2006)\end{array}$ & Continentale & Quantitatif & Faits avérés & Slow on-set & Interne \\
\hline $\begin{array}{l}\text { Rademacher- } \\
\text { Schulz et al. } \\
(2013)\end{array}$ & Communauté & $\begin{array}{l}\text { Méthode } \\
\text { mixte }\end{array}$ & Faits avérés & Slow on-set & Interne \\
\hline Sow et al. (2014) & Communauté & Qualitatif & Perception & Slow on-set & Interne \\
\hline Grolle (2015) & Continentale & Historique & Faits avérés & Slow on-set & Interne \\
\hline $\begin{array}{l}\text { Zickgraf et al. } \\
(2016)\end{array}$ & Continentale & Historique & Perception & Slow on-set & Interne \\
\hline $\begin{array}{l}\text { Zickgraf et al. } \\
(2016)\end{array}$ & Ville & Qualitatif & Faits avérés & Slow on-set & Interne \\
\hline Les températures & & & & & \\
\hline $\begin{array}{l}\text { Dillon et al. } \\
(2011)\end{array}$ & Régionale & Quantitatif & Faits avérés & Slow on-set & Interne \\
\hline Le couvert végétal & & & & & \\
\hline $\begin{array}{l}\text { Van der Geest et } \\
\text { al. (2010) }\end{array}$ & Pays & Quantitatif & Faits avérés & Slow on-set & Interne \\
\hline $\begin{array}{l}\text { Les migrations } \\
\text { internationales }\end{array}$ & & & & & \\
\hline Henry (2004b) & Pays & Quantitatif & Faits avérés & Slow on-set & $\begin{array}{l}\text { Interne et } \\
\text { internationale }\end{array}$ \\
\hline
\end{tabular}




\begin{tabular}{|c|c|c|c|c|c|}
\hline Naudé (2008) & Pays & Quantitatif & Faits avérés & Slow on-set & Internationale \\
\hline $\begin{array}{l}\text { Nawrotzki et al. } \\
(2016)\end{array}$ & Pays & Quantitatif & Faits avérés & Slow on-set & Internationale \\
\hline $\begin{array}{l}\text { Nawrotzki et } \\
\text { Bakhtsiyarava } \\
(2017)\end{array}$ & Pays & Quantitatif & Faits avérés & Slow on-set & Internationale \\
\hline $\begin{array}{l}\text { L'environnement } \\
\text { a un impact } \\
\text { modéré }\end{array}$ & & & & & \\
\hline $\begin{array}{l}\text { De Haan et al. } \\
(2002)\end{array}$ & Ménage & Qualitatif & Faits avérés & Slow on-set & Interne \\
\hline $\begin{array}{l}\text { Henry et al. } \\
(2004 a)\end{array}$ & Individus & Quantitatif & Faits avérés & Slow on-set & Interne \\
\hline Bleibaum (2009) & Pays & Qualitatif & Faits avérés & Slow on-set & Interne \\
\hline $\begin{array}{l}\text { Doevenspeck } \\
(2011)\end{array}$ & Pays & $\begin{array}{l}\text { Méthode } \\
\text { mixte }\end{array}$ & Perception & Slow on-set & Interne \\
\hline $\begin{array}{l}\text { Dreier et Sow } \\
(2015)\end{array}$ & Communauté & Qualitatif & Perception & Slow on-set & Interne \\
\hline $\begin{array}{l}\text { Codjoe et al. } \\
(2017)\end{array}$ & Communauté & Quantitatif & Faits avérés & Rapid on-set & Interne \\
\hline $\begin{array}{lr}\text { Les études } & \text { qui } \\
\text { tentent } & \text { de } \\
\text { mesurer } & \text { les } \\
\text { différents } & \\
\text { facteurs } & \end{array}$ & & & & & \\
\hline $\begin{array}{l}\text { Un phénomène } \\
\text { historique }\end{array}$ & & & & & \\
\hline Swift (1977) & Continentale & Historique & Faits avérés & Slow on-set & Internationale \\
\hline $\begin{array}{l}\text { Mortimore } \\
(1989)\end{array}$ & Continentale & $\begin{array}{l}\text { Méthode } \\
\text { mixte }\end{array}$ & Faits avérés & Slow on-set & $\begin{array}{l}\text { Interne et } \\
\text { internationale }\end{array}$ \\
\hline $\begin{array}{l}\text { Mounkaïla } \\
(2002)\end{array}$ & Régionale & Qualitatif & Faits avérés & Slow on-set & Interne \\
\hline
\end{tabular}




\begin{tabular}{|c|c|c|c|c|c|}
\hline $\begin{array}{l}\text { De Bruijn et Van } \\
\text { Dijk (2003) }\end{array}$ & Communauté & Qualitatif & Faits avérés & Slow on-set & Interne \\
\hline Roquet (2008) & Pays & $\begin{array}{l}\text { Méthode } \\
\text { mixte }\end{array}$ & Faits avérés & Slow on-set & Interne \\
\hline $\begin{array}{l}\text { Van der Geest } \\
(2009,2011)\end{array}$ & Régionale & Qualitatif & Faits avérés & Slow on-set & Interne \\
\hline Afifi (2011) & Pays & Qualitatif & Faits avérés & Slow on-set & $\begin{array}{l}\text { Interne et } \\
\text { internationale }\end{array}$ \\
\hline $\begin{array}{l}\text { Romankiewicz } \\
\text { et Doevenspeck } \\
(2015)\end{array}$ & Pays & $\begin{array}{l}\text { Méthode } \\
\text { mixte }\end{array}$ & Faits avérés & Slow on-set & Interne \\
\hline $\begin{array}{l}\text { Romankiewicz } \\
(2016)\end{array}$ & Communauté & Qualitatif & Faits avérés & Slow on-set & Interne \\
\hline $\begin{array}{l}\text { Delaunay et al. } \\
(2016)\end{array}$ & Communauté & $\begin{array}{l}\text { Méthode } \\
\text { mixte }\end{array}$ & Faits avérés & Slow on-set & Interne \\
\hline $\begin{array}{l}\text { Zickgraf et al. } \\
(2016)\end{array}$ & Communauté & Qualitatif & Faits avérés & Slow on-set & Interne \\
\hline $\begin{array}{l}\text { Impact de la } \\
\text { migration }\end{array}$ & & & & & \\
\hline Afifi (2009) & Pays & $\begin{array}{l}\text { Méthode } \\
\text { mixte }\end{array}$ & Faits avérés & Slow on-set & $\begin{array}{l}\text { Interne et } \\
\text { internationale }\end{array}$ \\
\hline $\begin{array}{l}\text { Failler et Binet } \\
(2010)\end{array}$ & Pays & Historique & Fait avérés & Slow on-set & $\begin{array}{l}\text { Interne et } \\
\text { internationale }\end{array}$ \\
\hline $\begin{array}{l}\text { L'environnement } \\
\text { et les facteurs } \\
\text { socio- } \\
\text { démographiques }\end{array}$ & & & & & \\
\hline Findley (1995) & Pays & Quantitatif & Faits avérés & Slow on-set & Interne \\
\hline Rain (1999) & Pays & Qualitatif & Faits avérés & Slow on-set & Interne \\
\hline $\begin{array}{lll}\text { Henry } & \text { et al. } \\
(2002) & & \end{array}$ & Pays & Quantitatif & Faits avérés & Slow on-set & Interne \\
\hline $\begin{array}{ll}\text { Purokayo et } \\
\text { Gabdo (2013) }\end{array}$ & Ville & Qualitatif & Perception & $\begin{array}{l}\text { Slow on-set et } \\
\text { rapid on-set }\end{array}$ & Interne \\
\hline
\end{tabular}




\begin{tabular}{|l|l|l|l|l|l|}
\hline $\begin{array}{l}\text { Van der Land et } \\
\text { Hummel (2013) }\end{array}$ & Pays & $\begin{array}{l}\text { Méthode } \\
\text { mixte }\end{array}$ & Faits avérés & Slow on-set & Interne \\
\hline Abu et al. (2014) & Ménage & Quantitatif & Faits avérés & Slow on-set & Interne \\
\hline $\begin{array}{l}\text { Ajaero et al. } \\
\text { (2016) }\end{array}$ & Ménage & $\begin{array}{l}\text { Méthode } \\
\text { mixte }\end{array}$ & Faits avérés & Rapid on-set & Interne \\
\hline $\begin{array}{l}\text { Approches } \\
\text { Globales }\end{array}$ & Pays & Quantitatif & Faits avérés & Slow on-set & Interne \\
\hline Hummel (2015) & Pays & Quantitatif & Faits avérés & Slow on-set & Interne \\
\hline $\begin{array}{l}\text { Liehr et } \\
\text { (2016) }\end{array}$ & Pl. & & & \\
\hline
\end{tabular}

\section{NOTES}

1. Par changements environnementaux nous entendons tous les écarts à la norme dans des domaines aussi différents que la pluviométrie, les températures, la fréquence des catastrophes, etc. qui ont un impact potentiel sur les conditions de vie des populations.

2. Dans ce présent article, nous avons volontairement utilisé une grille d'analyse large concernant les types de migration afin de garder un regard le plus exhaustif possible sur les études de cas présentées.

3. La première famine analysée se situe durant les années 1953 - 1954 alors que le niveau des précipitations était élevé ( $990 \mathrm{~mm}$ en 1952). La deuxième famine concerne les années 1972 - 1973 avec respectivement $329 \mathrm{~mm}$ et $290 \mathrm{~mm}$ de pluie. La dernière famine s'étale des années 1983 à 1985 avec une hauteur moyenne des précipitations de 387 mm en 1983 et 375 mm en 1984.

4. Il est possible de faire un parallèle avec une des conclusions de Beauchemin et Bocquier (2004). Ces auteurs examinent l'impact de la migration sur l'urbanisation de l'Afrique de l'Ouest francophone. Il en ressort que des nouveaux mouvements migratoires vers les zones rurales depuis les centres urbains apparaissent.

5. Il est à noter que cet aléa environnemental tout comme la montée des océans et l'érosion côtière sont peu étudiés en Afrique de l'Ouest et qu'ils méritent des recherches approfondies.

6. Voir tableau en annexe : « Aperçu des études de cas prises en compte».

\section{RÉSUMÉS}

Cet article fait le point des connaissances existantes sur le lien entre changements environnementaux et migrations en Afrique de l'Ouest. Les aspects liés aux variations pluviométriques, aux augmentations de températures, aux sécheresses, à l'élévation du niveau des mers sont en particulier abordés. Quarante-trois études de cas sont présentées ce qui 
représente de manière quasi exhaustive l'état de la recherche sur le thème. En se concentrant uniquement sur cette sous-région et en présentant les lieux d'études, les méthodes utilisées, les concepts mobilisés et les principaux résultats obtenus, cette revue de littérature a l'avantage d'exposer une vue d'ensemble détaillée de la recherche au niveau méthodologique, conceptuel et géographique. Nous mettons en évidence dix acquis et enseignements majeurs émergeant de la littérature et qui caractérisent les migrations environnementales en Afrique de l'Ouest et les qualifions avec les métriques éprouvées de l'Intergovernmental Panel on Climate Change (IPCC). Finalement, nous avançons que d'un point de vue théorique, les travaux doivent s'orienter vers une vision compréhensive des migrations qui placent les dégradations environnementales dans leur contexte socioéconomique et politique.

This paper reviews existing knowledge on the link between environmental changes and migration in West Africa. Aspects related to rainfall variations, temperature increases, droughts, and sea level rises are particularly addressed. Forty-three case studies are presented, which is almost an exhaustive representation of the state of research on the topic. By focusing only on this sub-region and presenting the places of study, the methods used, the concepts mobilized, and the main results obtained, this literature review has the advantage of presenting a detailed overview of the research at the methodological, conceptual and geographical level. We highlight ten major achievements and lessons emerging from the literature that characterize environmental migration in West Africa, qualified with the proven metrics of the Intergovernmental Panel on Climate Change (IPCC). Finally, we argue that from a theoretical point of view, work must be directed towards a comprehensive vision of migration that places environmental degradation in its socio-economic and political context.

\section{INDEX}

Mots-clés : changement climatique, changements environnementaux, migration, migrants environnementaux, Afrique de l'Ouest

Keywords : climate change, environmental changes, migration, environmental migrants, West Africa

\section{AUTEURS}

\section{LOIIC BRÜNING}

Assistant/doctorant, Université de Neuchâtel, Suisse, loic.bruening@unine.ch

\section{ETIENNE PIGUET}

Professeur, Université de Neuchâtel, Suisse, etienne.piguet@unine.ch 